\title{
Sequential MRI studies in patients with cervical cord injury but without bony injury
}

\author{
K Shimada and T Tokioka \\ Department of Orthopaedic Surgery, Okayama, Rousai Hospital, Midorimachi 702, Okayama City, Japan
}

Sequential magnetic resonance imaging (MRI) of 23 patients who suffered cervical spinal cord injury without bony injury was performed prospectively. The major cord injury detected by MRI was at the C3-4 disc level in 16 patients (70\%). Three patterns of signal changes were observed. Enhancement of the injured cord was observed on Gd-DTPAenhanced MRI in 10 patients and the palsy of these patients was more severe than that of those without enhancement. Enhancement was recognised about 2 weeks earlier than the signal change (from isointense to low intensity) on T1-weighted images. This enhancement might represent necrosis, absorption, and reorganisation of the spinal cord, and appears to be a sign of a poor prognosis or an indication that damage is permanent. Another characteristic imaging finding was a vague high intensity signal in the dorsal column of the spinal cord extending rostrally from the main lesion, which appeared 2-3 months after injury and disappeared around 6 months. This finding represents Wallerian degeneration of the corticospinal tract in the cervical cord. Rigidity of the legs and sensory changes of the fingers became more prominent during this period.

Keywords: cervical spinal cord injury; magnetic resonance imaging; bone injury

\section{Introduction}

The location, extent, and severity of traumatic cervical spinal cord injury without bony injury can be well delineated by MRI, which also reflects the histopathological features of intramedullary lesions.

There are several reports on the MRI findings in patients who have suffered spinal cord injury associated with fracture and dislocation of the cervical spine, but there have been few serial MRI studies of spinal cord injury without bony injury. We performed serial MRI from the acute to the chronic stage in patients who had cervical cord injury without bony injury and determined the characteristic MRI findings as well as the sequential changes of signal intensity. We also assessed the relationships between the MRI findings and the histopathological changes of the spinal cord or the clinical features.

\section{Materials and methods}

Twenty-three patients who had acute traumatic cervical cord injury without bony injury were studied. There were 22 men and one woman. The age at injury ranged from 25 to 89 years (mean: 62.4 years). Paralysis at the time of injury was grade A in three patients, grade B in 11 , grade $C$ in six, and grade $D$ in three according to Frankel's classification. None of the patients had a fracture or a dislocation of the cervical spine and all were treated conservatively. The first MRI study was performed within $48 \mathrm{~h}$ of injury, and subsequent stud- ies were done after approximately $2-3$ weeks, $2-3$ months, and 6 months. Enhanced imaging with GdDTPA was performed in 13 patients. Images were obtained using a 1.5 tesla superconducting MR scanner with a surface coil. T1-weighted images were obtained with an echo time (TE) of $17 \mathrm{~ms}$ and a pulse repetition time (TR) of $550 \mathrm{~ms}$, and T2-weighted images were obtained with a TE of $130 \mathrm{~ms}$ and a TR of $3200 \mathrm{~ms}$. The spine-echo (SE) technique was used and the slice thickness was $5 \mathrm{~mm}$.

\section{Results}

Relationship of cord injury on MRI to cervical spondylosis

The major spinal cord injury detected by MRI was at the $\mathrm{C} 3-4$ disc level in 16 patients $(70 \%)$, at $\mathrm{C} 4-5$ in four, and at C5-6 and C6-7 in one case each. The main site of cord damage corresponded to the narrowest part of the spinal canal in nine patients with cervical spondylosis, whereas there was no relationship between the site of cord damage and the narrowest part of the canal in 10 patients. The other four patients had no degenerative changes of the cervical spine.

Characteristic MRI findings, serial changes, and correlation with the clinical features (Figure 1) We classified the patients into three groups depending on the changes of signal intensity on serial MRI. Group 
Group 1 ( 2 cases) (T1W images-iso, T2W images-iso)

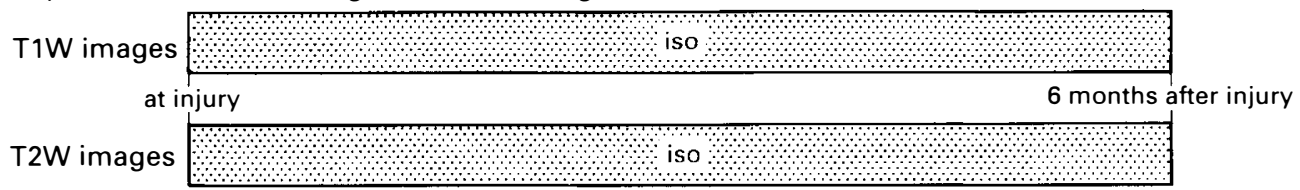

Group 2 (9 cases) (T1W images-iso, T2W images-high $\rightarrow$ iso)

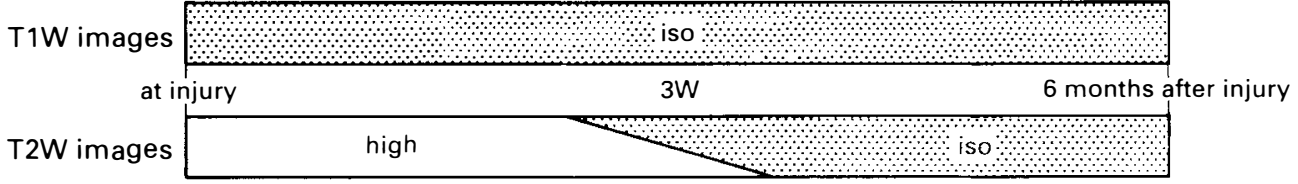

Group 3 (12 cases) (T1W images-iso-low, T2W images-high)

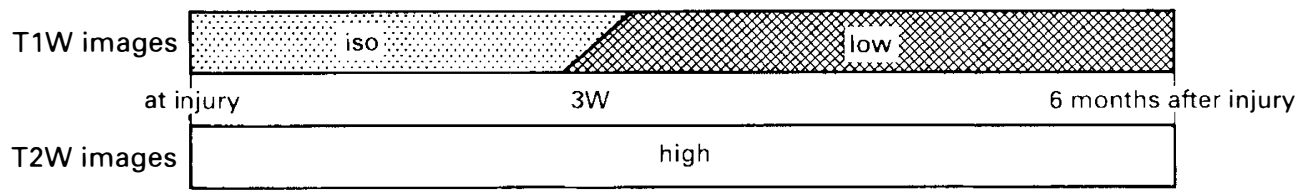

Figure 1 Characteristic MRI finding and its serial changes. The patients were classified into three groups depending on the pattern of signal changes. Group 1 showed no signal changes (iso-intensity) on both T1-weighted and T2-weighted images throughout the clinical course. Group 2 showed no signal changes on T1-weighted images, but T2-weighted images showed high to iso-intensity change from the acute to chronic stages. T1-weighted images of group 3 showed an iso to low-intensity change from the acute to chronic stages, and T2-weighted images showed a high signal intensity throughout the clinical course

1 was patients with no signal changes on both T1weighted and $\mathrm{T} 2$-weighted images throughout their clinical course. The patients in group 1 showed no motor palsy, except for weakness of hand grip, but complained of numbness and dysaesthesia of the arms and fingers. These symptoms all resolved completely within 2 months of injury (Figure 2).

In group 2, there were no signal changes on T1-weighted images from the acute to the chronic stage but T2-weighted images showed a high signal intensity area in the acute stage that gradually became limited to the main site of damage and almost completely disappeared from 3-6 weeks after injury (Fig 3). The patients in group 2 showed a palsy of the central cord type. Their motor paralysis recovered well, but there was residual finger dysfunction in eight out of nine cases. One patient showed complete motor recovery and only slight numbness of the finger tips.

In group 3, there were no signal changes on $\mathrm{T} 1$ weighted images at the time of injury, but a circumscribed low intensity area appeared 4-6 weeks after injury. An extensive high signal intensity area was observed from the acute to the chronic stage on T2-weighted images, but this area decreased in size and become clearer around 4 weeks after injury (Fig 4). The clinical features of this group were more severe when compared to the other groups. Four patients had central cord palsy and eight had transverse type palsy (five incomplete and three complete). The pattern of neurological recovery was as follows: group 1 : D-E, two cases; group 2: B-D, three cases; $\mathrm{C}-\mathrm{D}$, two cases; $\mathrm{D}-\mathrm{E}$, one case; group 3: $\mathrm{A}-\mathrm{A}$, three cases; $\mathrm{B}-\mathrm{C}$, one case; $\mathrm{B}-\mathrm{B}$, one case; $\mathrm{B}-\mathrm{D}$, two cases; $\mathrm{C}-\mathrm{C}$, five cases.

\section{T1-weighted Gd-DTPA- enhanced MRI findings (Figure 5)}

Enhanced MRI studies were performed serially in 13 patients and enhancement was observed around 2 weeks after injury in 10 patients. This enhancement subsequently decreased gradually and disappeared by 2-3 months after injury. No enhancement was observed in three patients and they had less severe palsy than the others.

\section{High signal intensity of the dorsal column on chronic} T2-weighted images

T2-weighted images showed a continuous slightly high signal intensity line in six patients corresponding to the dorsal column and extending rostrally from the main lesion at 2-3 months after injury. This high signal intensity change became obscure and disappeared at around 6 months after injury (Figure 6). All six patients were in group 3 and had transverse palsy (complete in two and incomplete but severe in four). Rigidity of the legs and sensory changes of the fingers increased to some degree from the time when the signal change was observed in these six patients. 


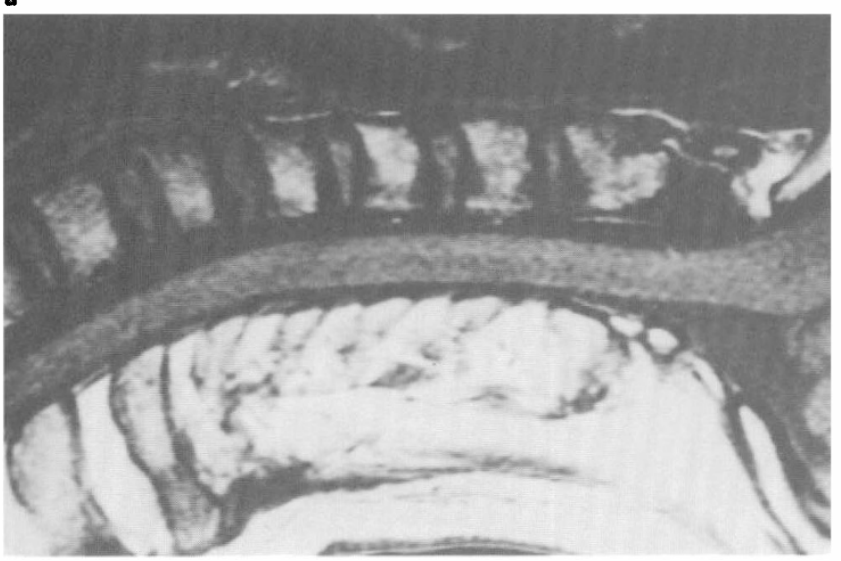

b

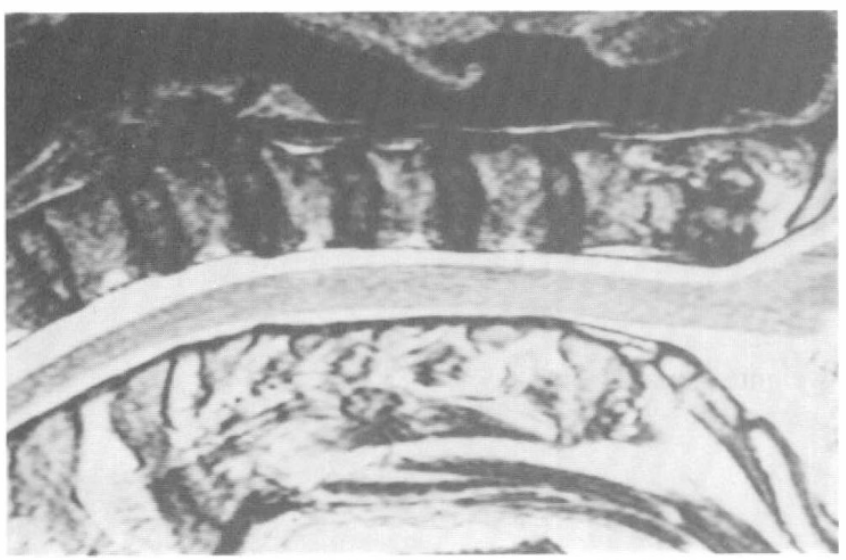

Figure 2 A 45-year-old man suffered cervical spinal cord injury but without bony injury. Paralysis was Frankel grade $\mathrm{D}$ in the acute stage and recovered completely by 2 months after injury. (a) T1-weighted images at $24 \mathrm{~h}$ after injury. (b) T2-weighted images at $24 \mathrm{~h}$ after injury. No signal changes were observed throughout the clinical course

\section{Discussion}

Several authors have described the MRI findings of spinal cord injury associated with bony injury and have discussed the relationship between the histopathological changes and the MRI features. ${ }^{1-3}$ In clinical practice, making a precise diagnosis of the main site of spinal cord damage and predicting the outcome in patients who have cervical cord trauma without bony injury is very difficult. Accordingly, we performed MRI studies in such patients sequentially for several months after the onset of injury.

One interesting finding was the location of the main site of spinal cord injury. The mechanism of injury in the patients of the present series seemed to be hyperextension. The main site of spinal cord injury was the C3-4 disc level in 16 patients $(70 \%)$ and there was no correlation between the site of injury and that of the most marked degeneration of the cervical spine (the narrowest part of the canal) in 10 patients with cervical spondylosis. Although this series was small, we should a

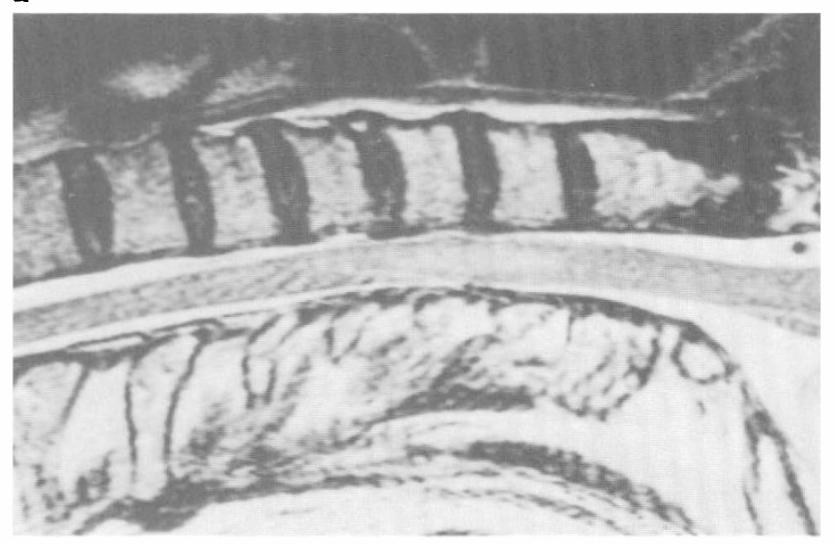

b

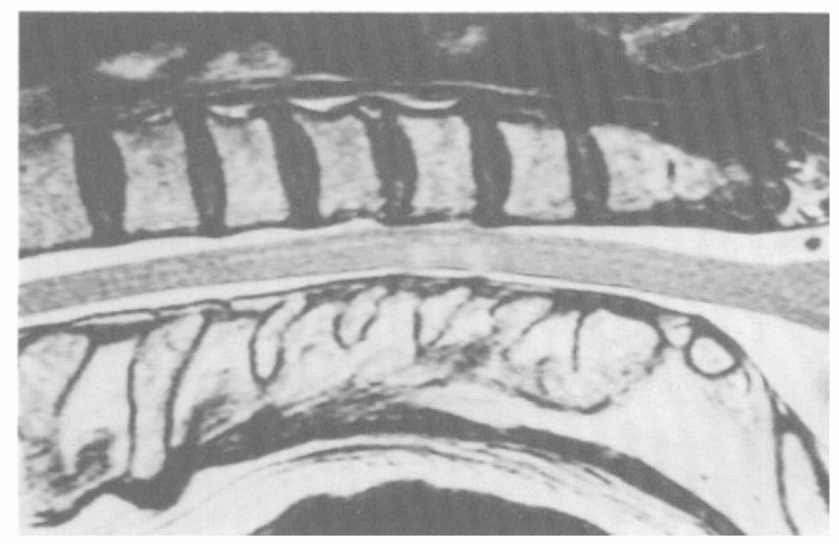

c
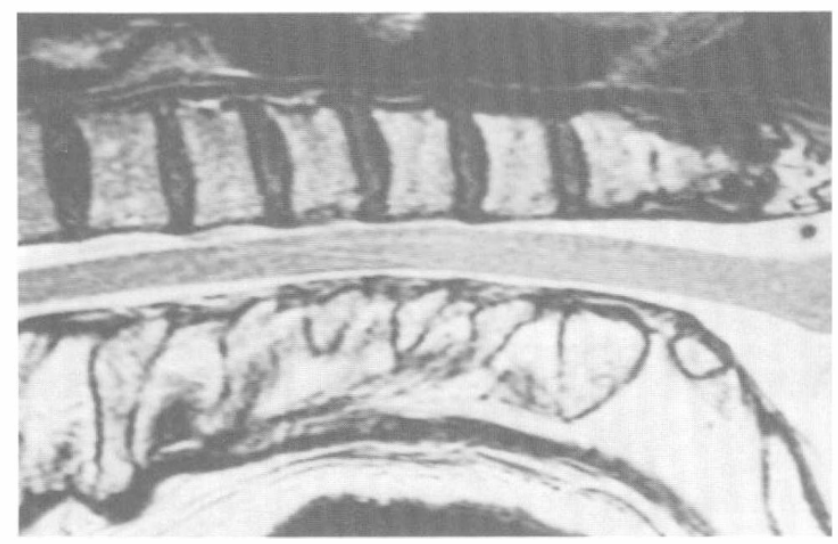

Figure 3 A 64-year-old man suffered cervical spinal cord injury without bony injury. Paralysis was grade $\mathrm{C}$ in the acute stage and recovered to grade $D$ by 12 months after injury. (a) T2-weighted images at $48 \mathrm{~h}$ after injury. Vague high signal intensity at $\mathrm{C} 3-4$. (b) T2-weighted images at 3 weeks after injury. The high signal intensity area is clearer and has decreased in size at $\mathrm{C} 3$. The thin line of high signal intensity might indicate the central grey commisure. (c) T2-weighted images at 6 weeks after injury. The high signal intensity has almost disappeared.

keep these facts in mind when evaluating patients and planning the management of this type of injury.

The basic MRI findings were that there was no change of signal intensity on T1-weighted images and a 


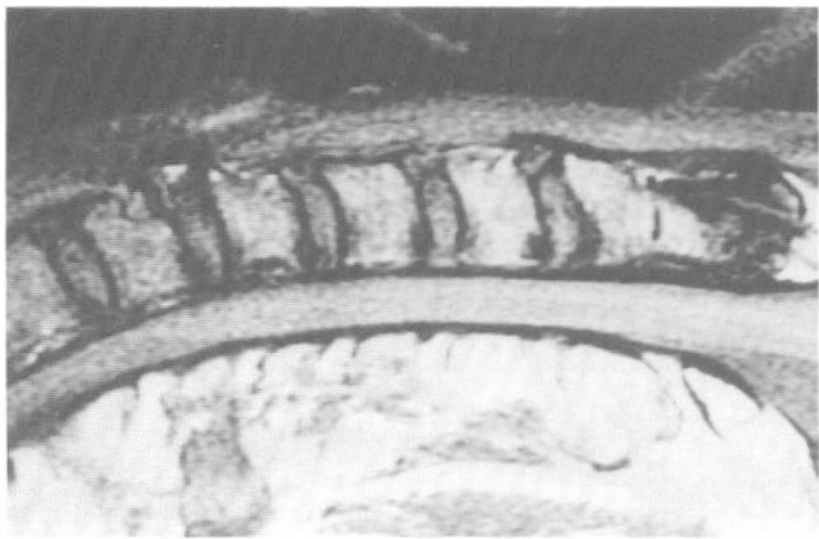

b

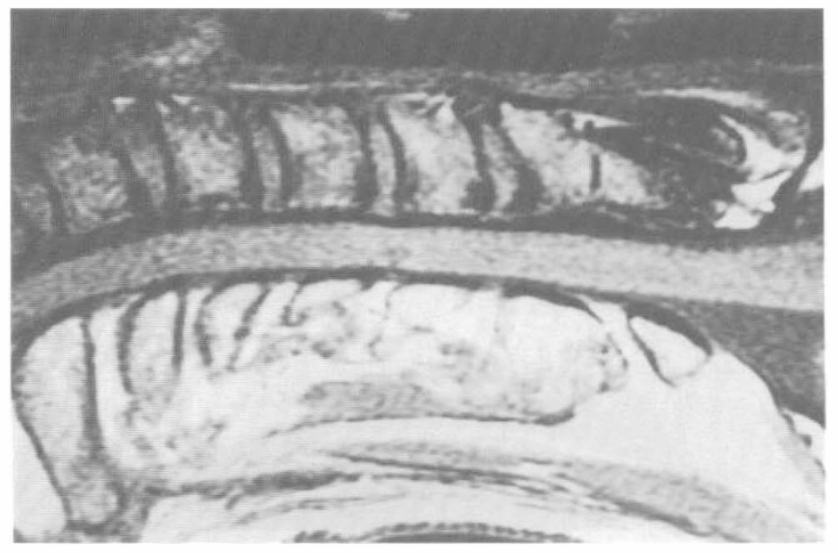

$c$

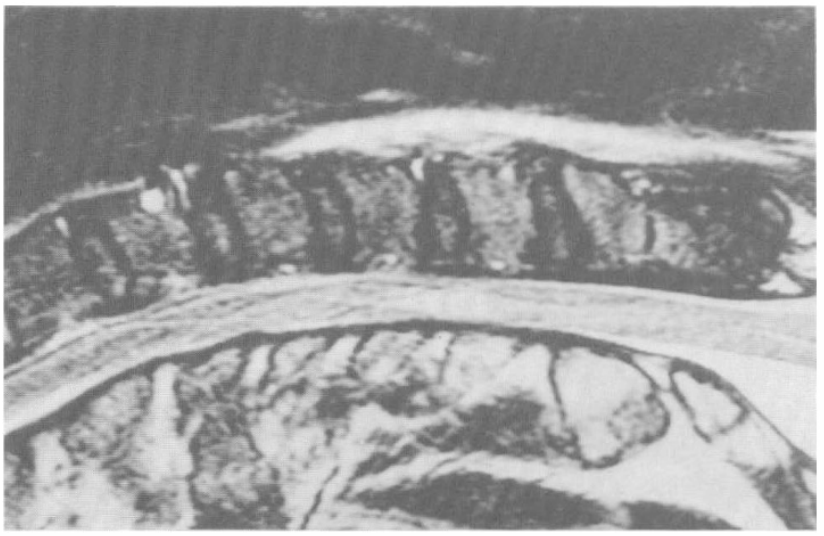

high signal intensity area on T2-weighted images in the acute stage, with a well-defined low intensity area on T1-weighted images and a high intensity area on T2-weighted images in the subacute as well as chronic stages. With reference to the results of other investigators $^{4-6}$ on the histopathological features of spinal cord injury, it is speculated that the acute signal changes represent oedema of the spinal cord, while the subacute and chronic changes represent necrosis, myelomalacia, spongiform degeneration, and reorganisation of the grey matter. A low signal intensity on T2-weighted images in the acute stage and a high signal intensity area on T1-weighted images in the subacute stage have

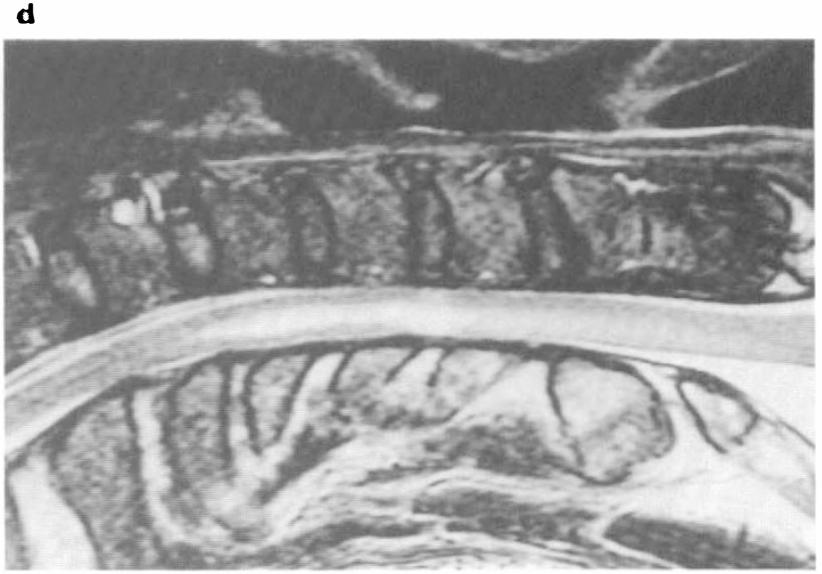

$\mathbf{e}$

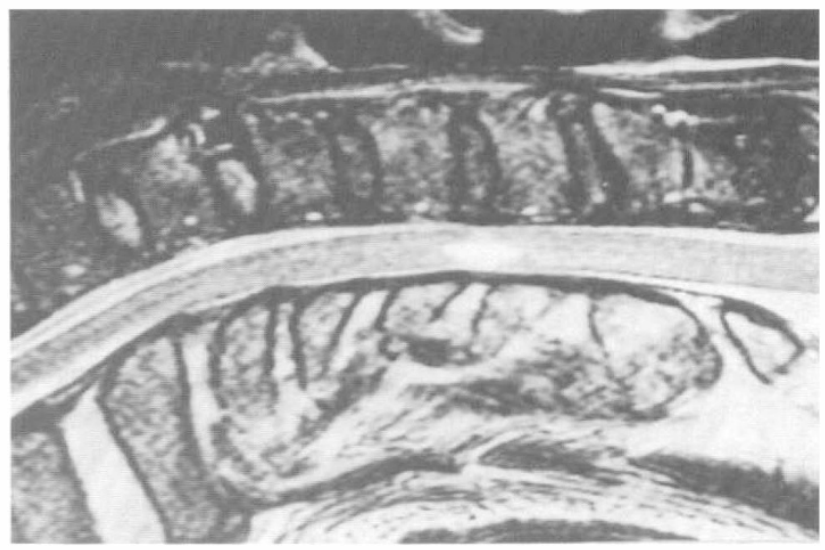

Figure 4 A 35-year-old man suffered cervical spinal cord injury without bony injury. Paralysis was grade B in the acute stage and recovered to grade $D$ by 12 months after injury. (a) T1-weighted images at $14 \mathrm{~h}$ after injury. No signal change was observed. (b) T1-weighted images at 6 weeks after injury. A circumscribed low intensity area was observed at the C3-4 disc level. (c) T2-weighted images at $14 \mathrm{~h}$ after injury. An extensive and vague high signal intensity area was centered at the C3-4 disc level. Artefacts are prominent. (d) T2-weighted images at 13 days after injury are clearer than those in (c). (e) T2-weighted images at 4 weeks after injury. A clear and well defined high signal intensity at the $\mathrm{C} 3-4$ disc level

been reported as indicating a poor prognosis.,7-8 However, no patient (even with complete paralysis) showed these signal changes in our present series. There has been some speculation that these signal changes indicate the metabolism of haematoma. ${ }^{1,9}$ The traumatic force at the time of injury might have been smaller in our patients because they had no bone damage, thus intramedullary bleeding may have been less severe than may occur after a spinal cord injury associated with bony injury, and this may be why such signal changes were not observed in our series.

There have only been a few reports on enhanced MRI studies of traumatic spinal cord injury ${ }^{4,10}$ and 


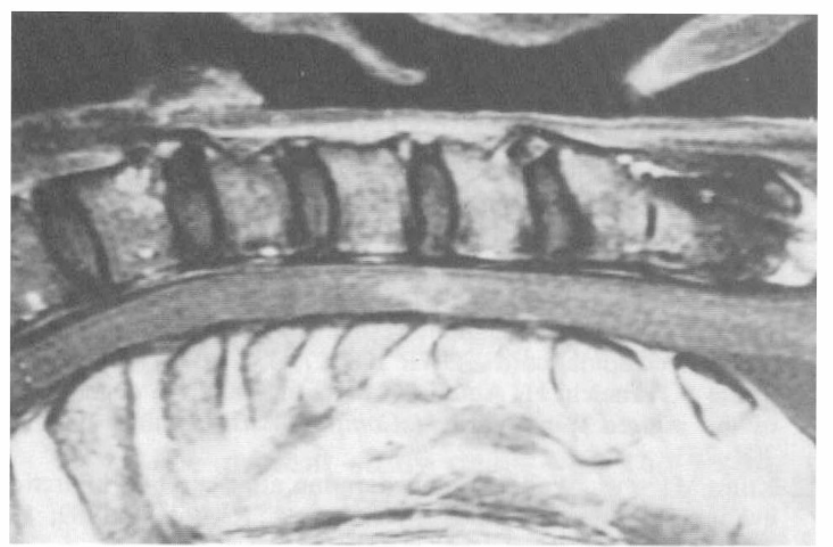

b

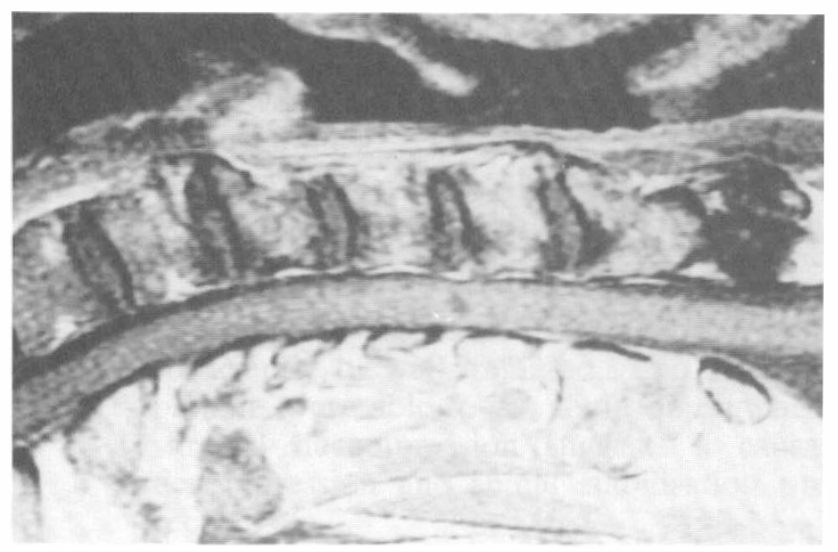

Figure 5 The 35-year-old man shown in Figure 4. (a) T1weighted enhanced images at 2 weeks after injury. Vague enhancement at the C3-4 disc level. (b) The same study at 4 weeks after injury shows no enhancement

there appears to be some controversy as to its value. In our series, enhancement at the site of cord damage was observed around 2 weeks after injury and then decreased gradually to disappear by $2-3$ months. Usually, this enhancement was recognised before a low signal intensity area appeared on T1-weighted images. Thus, it might have represented the early process of necrosis, absorption, and reorganisation of the damaged spinal cord and may be a sign of a poor prognosis or an indication that damage to the cord is permanent.

Another interesting finding was a high intensity signal in the dorsal column spreading rostral from the main injury site on T2-weighted images in the chronic stage. There is little mention of such a signal change after traumatic spinal cord injury in the literature. ${ }^{5,11}$ Taking into consideration other reports that are available and the MRI study of cerebral infarction by Kuhn et $a l,{ }^{12}$ this signal change is considered likely to represent Wallerian degeneration of the corticospinal tract in the cervical cord. Kuhn classified Wallerian degeneration into four stages in his MRI study of cerebral lesions; a low signal intensity was seen in stage 2 , with a sudden change to a high signal intensity on
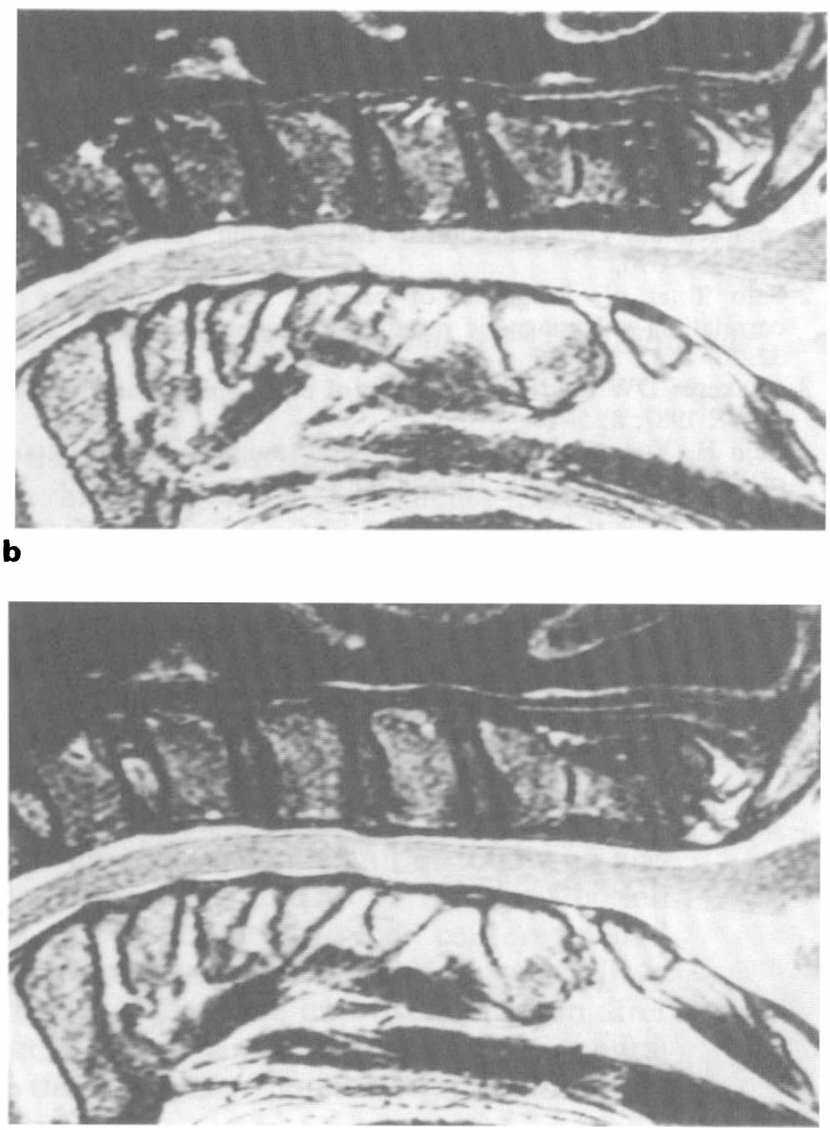

Figure 6 The 35-year-old man shown in Figure 4. (a) T2weighted images at 3 months after injury. High signal intensity extends rostral from the main lesion. (b) T2weighted images at 6 months after injury. The long high signal intensity has almost disappeared

T1-weighted images in stage 3 . In our series, only a high signal was observed at 2-3 months after injury and this may correspond to stage 3 Wallerian degeneration in Kuhn's classification. One of the interesting clinical manifestations was an increase of rigidity and sensory changes at this time.

We conclude that a more precise diagnosis and more effective treatment can be achieved by utilising MRI in patients who have traumatic cervical spinal cord injury without bony injury.

\section{Conclusions}

Sequential MRI was performed in 23 patients who had traumatic cervical spinal cord injury without bony injury. The main site of cord injury was at the C3-4 disc level in 16 patients $(70 \%)$. The basic MRI findings were no change of signal intensity on T1-weighted images and a high signal intensity area on T2-weighted images in the acute stage, as well as a well defined low intensity area on T1-weighted images and a high intensity area on T2-weighted images in the subacute and chronic 
stages. MRI findings that may indicate Wallerian degeneration were also detected.

\section{References}

1 Hackney DB et al. Hemorrhage and edema in acute spinal cord compression: Demonstration by MR imaging. Radiology 1986; 161: $387-390$.

2 Sato $\mathrm{T}$ et al. Prognosis of cervical spinal cord injury in correlation with magnetic resonance imaging. Paraplegia 1994; 32: $81-85$.

3 Chakeres DW et al. MR imaging of acute spinal cord trauma. AJNR 1987; 8: 5-10.

4 Fujii H, Yone K, Sakou T. Magnetic resonance imaging study of experimental acute spinal cord injury. Spine 1993; 18: 2030-2034.

5 Ohshio I et al. Correlation between histopathologic features and magnetic resonance images of spinal cord lesion. Spine 1993; 18: 1140-1149.

6 Tanaka J, Shingu H. Neuropathology of spinal cord injury and its pathogenesis. Rinsho Seikei Geka 1991; 26: 1137-1144.

7 Bondurant FJ et al. Acute spinal cord injury: a study using physical examination and magnetic resonance imaging. Spine 1991; 15: 161-168.

8 Mori A et al. Magnetic resonance imaging of cervical cord injury. Rinsho Seikei Geka 1991; 26: 1163-1171.

9 Gmori JM et al. Intracranial hematomas: imaging by high-field MR. Radiology 1985; 157: 87-93.

10 Quencer RM et al. Magnetic resonance imaging of the chronically injured spinal cord. AJNR 1986; 7: 457-464.

11 Terae S, Taneichi H, Abumi K. MRI of Wallerian degeneration of the injured spinal cord. J Comput Assist Tomogr 1993; 17: 700-703.

12 Kuhn MJ et al. Wallerian degeneration after cerebral infarction: evaluation with sequential MR imaging. Radiology 1989; 172: 179-182. 